\title{
Synchronization of Time-Continuous Chaotic Oscillators
}

\author{
Yanchuk, S.; Maistrenko, Yuri; Mosekilde, Erik
}

Published in:

Chaos

Link to article, DOI:

$10.1063 / 1.1496536$

Publication date:

2003

Document Version

Publisher's PDF, also known as Version of record

Link back to DTU Orbit

Citation (APA):

Yanchuk, S., Maistrenko, Y., \& Mosekilde, E. (2003). Synchronization of Time-Continuous Chaotic Oscillators. Chaos, 13(1), 388-400. https://doi.org/10.1063/1.1496536

\section{General rights}

Copyright and moral rights for the publications made accessible in the public portal are retained by the authors and/or other copyright owners and it is a condition of accessing publications that users recognise and abide by the legal requirements associated with these rights.

- Users may download and print one copy of any publication from the public portal for the purpose of private study or research.

- You may not further distribute the material or use it for any profit-making activity or commercial gain

- You may freely distribute the URL identifying the publication in the public portal

If you believe that this document breaches copyright please contact us providing details, and we will remove access to the work immediately and investigate your claim 


\title{
Synchronization of time-continuous chaotic oscillators
}

\author{
Sergiy Yanchuk ${ }^{\mathrm{a})}$ and Yuri Maistrenko \\ Institute of Mathematics, National Academy of Sciences of Ukraine, Kiev 01601, Ukraine \\ Erik Mosekilde \\ Center for Chaos and Turbulence Studies, Technical University of Denmark, 2800 Kgs. Lyngby, Denmark
}

(Received 25 February 2002; accepted 21 May 2002; published 21 February 2003)

\begin{abstract}
Considering a system of two coupled identical chaotic oscillators, the paper first establishes the conditions of transverse stability for the fully synchronized chaotic state. Periodic orbit threshold theory is applied to determine the bifurcations through which low-periodic orbits embedded in the fully synchronized state lose their transverse stability, and the appearance of globally and locally riddled basins of attraction is discussed, respectively, in terms of the subcritical, supercritical nature of the riddling bifurcations. We show how the introduction of a small parameter mismatch between the interacting chaotic oscillators causes a shift of the synchronization manifold. The presence of a coupling asymmetry is found to lead to further modifications of the destabilization process. Finally, the paper considers the problem of partial synchronization in a system of four coupled Rössler oscillators. (C) 2003 American Institute of Physics. [DOI: 10.1063/1.1496536]
\end{abstract}

The collective behavior of systems of coupled nonlinear oscillators is of significant interest in many areas of science and technology. It is likely, for instance, that transitions between different states of synchronization among the cells or functional units can play a major role in the regulation of many physiological systems. In the field of macroeconomics, synchronization phenomena may explain how the oscillatory dynamics of individual sectors or industries, rather than being averaged out in the aggregate, can lock together and contribute to the formation of the characteristic business and investment cycles. Particularly interesting is the case where the individual oscillators behave chaotically. In the present paper, we use a system of coupled Rössler oscillators to review some of the basic aspects of the theory of full synchronization for time-continuous chaotic oscillators. At the end of the paper we discuss the so-called cluster formation process by which the fully synchronized chaotic state loses its stability and breaks up into groups of oscillators with different dynamics, but such that the oscillators within a given group maintain synchrony.

\section{INTRODUCTION}

In the early 1980s, Fujisaka and Yamada ${ }^{1}$ showed how two identical chaotic oscillators under variation of the coupling strength can attain a state of complete synchronization in which the motion of the coupled system takes place on an invariant subspace of phase space, the synchronization manifold. This type of chaotic synchronization has subsequently been studied by a significant number of investigators, and a variety of applications for chaos suppression, for monitoring and control of dynamical systems, and for different communication purposes have been suggested. ${ }^{2,3}$ In addition to vari-

\footnotetext{
${ }^{a)}$ Electronic mail: yanchuk@imath.kiev.ua
}

ous electronic systems, ${ }^{4,5}$ synchronization of identical chaotic oscillators has also been investigated for laser systems, ${ }^{6}$ for coupled superconducting Josephson junctions, ${ }^{7}$ and for interacting electrochemical reactors. ${ }^{8}$

Similar phenomena are of interest in connection with a variety of different biological ${ }^{9,10}$ and economic ${ }^{11}$ systems. It has long been recognized, for instance, that the insulin producing $\beta$ cells of the pancreas produce complicated patterns of spikes and bursts in their membrane potentials, ${ }^{12}$ and these dynamics may also become chaotic. ${ }^{13}$ The $\beta$ cells interact with one another via the diffusive exchange of ions and small molecules through gap junctions, and, as experiments show, ${ }^{14}$ this interaction can lead to synchronization of larger groups of cells in an islet of Langerhans. In the field of macroeconomics, individual sectors and industries interact via the exchange of goods and units of capital, and this interaction may cause the industries to synchronize their individual commodity and investment cycles so as to produce the well-known business cycles. ${ }^{15}$

For systems of three (or more) coupled identical chaotic oscillators, one can observe the phenomenon of partial synchronization ${ }^{16}$ where some of the oscillators synchronize while others do not. This phenomenon is again of interest in connection with the development of various communication systems. In the biological systems one often encounters large ensembles of interacting cells or functional units, each displaying complicated nonlinear dynamic phenomena. With dominating local (i.e., nearest-neighbor) coupling, such ensembles tend to display various forms of traveling waves and spatiotemporal chaos. ${ }^{17}$ With dominant global coupling, on the other hand, i.e., when each oscillator is coupled to a mean field produced by the average dynamics of the whole ensemble of oscillators, one can observe the phenomenon of clustering. ${ }^{18,19}$ The fully synchronized state splits up into groups of oscillators such that the oscillators within each group maintain synchrony, but there is no synchronization between the groups. 
An important question that arises in connection with the synchronization of identical chaotic oscillators concerns the form of the basin of attraction for the fully synchronized chaotic state, i.e., for which initial conditions will the oscillators be able to synchronize if they are started out of synchrony? Other important questions relate to the stability of the synchronized state to a small parameter mismatch between the interacting oscillators and to the behavior of the coupled system, once the synchronization breaks down. Studies of these and related phenomena have led to the discovery of variety of new phenomena, including riddled basins of attraction, ${ }^{20}$ soft and hard riddling bifurcations, ${ }^{21}$ and on-off intermittency. ${ }^{22-24}$ Also interesting is the particular form of chaos-hyperchaos transition ${ }^{25,26}$ that can occur in connection with the loss of complete synchronization.

The purpose of the present paper is to illustrate some of the basic phenomena that one can observe in connection with chaotic synchronization in time-continuous systems. Our presentation is based on a series of recent publications in which we have investigated riddling and blowout bifurcations in systems of coupled Rössler oscillators ${ }^{27-29}$ and coupled pancreatic $\beta$ cells. ${ }^{30}$ Periodic orbit threshold theory $^{31,32}$ is applied to a system of two coupled Rössler oscillators in order to determine the bifurcations through which low-periodic orbits embedded in the fully synchronized chaotic state lose their transverse stability. The appearance of locally and globally riddled basins of attraction is discussed, respectively, in terms of the subcritical, supercritical nature of the riddling bifurcations in which the embedded in the synchronized chaotic state orbit loses its transverse stability. We show how the introduction of a small parameter mismatch between the interacting oscillators and of a coupling asymmetry modifies the riddling bifurcation. The significance of other stable states in the vicinity of the synchronized chaotic state is discussed, and the paper also presents some preliminary results on clustering in systems of many coupled chaotic oscillators.

\section{TRANSVERSE STABILITY OF THE SYNCHRONOUS ATTRACTOR}

We consider a system of two coupled oscillators of the form

$$
\frac{d x}{d t}=f(x)+C(y-x), \quad \frac{d y}{d t}=f(y)+C(x-y) .
$$

Here $x$ and $y$ represent the state variables for the oscillators, and the matrix $C$ stands for the coupling. The nonlinear function $f$ determines the dynamics of the individual system without coupling. For simplicity, we consider a diagonal coupling matrix $C=\operatorname{diag}\left\{d_{1}, d_{2}, d_{3}\right\}$, where $d_{1}, d_{2}$, and $d_{3}$ are parameters measuring the coupling strength.

Although system (1) has a relatively simple form, it incorporates important features of a large class of coupled oscillator systems and, as we will show, it manifests a great variety of complex behaviors. Hence, Eq. (1) can be regarded as a model for the qualitative description of many different phenomena that arise in coupled oscillator systems.

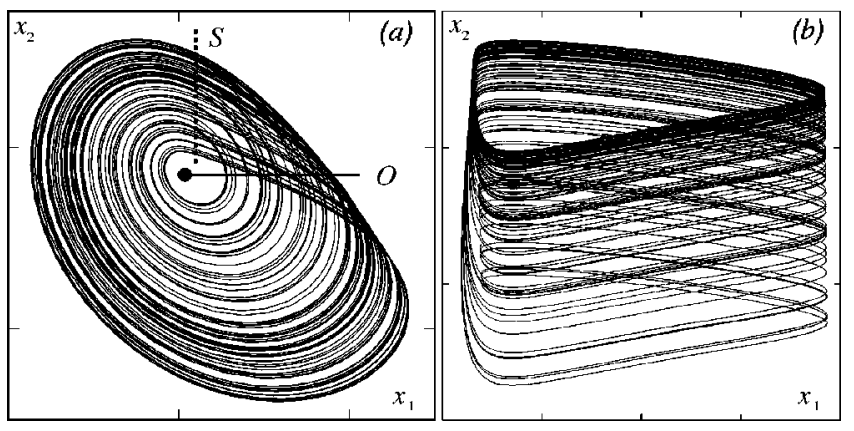

FIG. 1. Typical attractors of the Rössler system (a) and of the pancreatic $\beta$-cell model (b). In (a), $O$ denotes the unstable equilibrium point, and $S$ is the Poincare section used in connection with Fig. 12.

An important feature of system (1) is that the hyperplane $x=y$ is invariant. This set is usually called the synchronization manifold. This invariance implies that if at a given time $t_{1}$ the states of the two oscillators coincide $x\left(t_{1}\right)=y\left(t_{1}\right)$, then they will coincide for all subsequent moments $x(t)$ $=y(t)$. Within the synchronization manifold, the motion is described by the equation for the single oscillator $\dot{x}=f(x)$.

If we consider models of real-world systems, it is natural to assume that there exists an attracting set $\mathcal{A}$ for the single uncoupled oscillator, i.e., $x(t) \rightarrow \mathcal{A}$ with $t \rightarrow \infty$ for all initial conditions from some neighborhood of $\mathcal{A}$. We confine our analysis to the case where the set $\mathcal{A}$ is chaotic. As a specific example, we consider a Rössler system described by the following right-hand side:

$$
f=\left(\begin{array}{c}
-x_{2}-x_{3} \\
x_{1}+a x_{2} \\
b+x_{3}\left(x_{1}-c\right)
\end{array}\right)
$$

with the constants $a=0.42, b=2.0$, and $c=4.0$. An illustration of how similar approaches work for a model of chaotically spiking pancreatic $\beta$ cells may be found in our papers. ${ }^{29,30}$ In particular, we have studied the effects of a parameter mismatch on the synchronization. We have also determined the regions in the parameter space where the fully synchronized state is asymptotically, respectively weakly, stable. The $\beta$-cell model ${ }^{33}$ is a stiff system of differential equations in $R^{3}$. The attractors of the corresponding individual oscillators are shown in Fig. 1(a) for the Rössler oscillator and in Fig. 1(b) for the biological cell model, respectively.

In both cases the synchronization manifold of the coupled system (1) contains a chaotic attractor $A=\{(x, y): x$ $=y \in \mathcal{A}\}$. The stability properties of this attractor constitute the main problem of our investigation. In the following, we will refer to $A$ as the synchronous attractor. We will say that the system becomes synchronized if the set A is asymptotically stable, i.e., if $(x, y) \rightarrow A$ with $t \rightarrow \infty$ for some open set of initial conditions $U$ in the full phase space. As we shall see, our coupled oscillator system can become synchronized for a sufficiently strong coupling. If the condition $\|x(t)-y(t)\|$ $\rightarrow 0$ as $t \rightarrow \infty$ holds for all initial conditions from $U=R^{6}$, then we have global identical synchronization. Strictly speaking, the above-given definition depends on the choice of the attractor $\mathcal{A}$. In the case of multistability for the indi- 
vidual oscillator, the situation may arise where one synchronous attractor is stable while, for the same coupling, another is not.

The largest transverse Lyapunov exponent $\lambda_{\perp}$ provides a measure of the average stability of the synchronized chaotic state with respect to perturbations perpendicular to the synchronization manifold. As long as $\lambda_{\perp}$ is negative, the synchronized state is at least weakly stable. ${ }^{34}$ The transition in which $\lambda_{\perp}$ becomes positive is referred to as the blowout bifurcation. This transition may lead to an abrupt loss of stability for the synchronized chaotic state. Alternatively, one may observe the interesting form of bursting behavior known as on-off intermittency. Immediately after the blowout bifurcation, as long as $\lambda_{\perp}$ is still relatively small, a trajectory started near the synchronized chaotic state may spend a long time in the neighborhood of this state. However, sooner or later the repelling character of the synchronized state manifests itself, and the trajectory exhibits a burst in which it moves far out in phase space. Provided that the trajectory does not "find" another limiting state or diverges to infinity, after some time it will return to the neighborhood of the synchronized state, and the process will continue to repeat itself in an apparently random manner. On-off intermittency is distinguished from other, more conventional, forms of intermittency by the fact that the laminar phase is chaotic.

Riddled basins of attraction may be observed on the other side of the blowout bifurcation where $\lambda_{\perp}<0$. Even though the synchronized chaotic state is now attracting on average, particular orbits embedded in this state may be transversely unstable. As a result one can observe a situation where the synchronized chaotic state attracts a positive Lebesgue measure set of points from its vicinity. Arbitrarily close to any such point, however, there will be a positive Lebesgue measure set of points that are repelled from the synchronized state. The transition in which the first orbit on the synchronized state loses its transverse stability is referred to as the riddling bifurcation. As we shall show, this bifurcation may be either sub- or supercritical in nature, and the type of criticality has a significant influence on the character of the riddling behavior.

The fact that the synchronization manifold has a simple geometrical structure (i.e., a hyperplane) gives us an easy way to introduce a coordinate system that can separate the state variables into the longitudinal $\eta=x+y$ coordinates and the coordinates $\xi=x-y$ transverse to the synchronization manifold. Then for all synchronous motions we have $\xi=0$ and

$$
x(t)=y(t)=s(t), \quad \dot{s}=f(s), \quad \eta(t)=2 s(t) .
$$

Stability of the attractor $A$ within the synchronization manifold follows from the stability of the attractor $\mathcal{A}$ for the individual oscillator. Hence, the main problem is reduced to analyzing the stability of the synchronous attractor in the transverse directions $\xi$. The local analysis for transverse perturbations $\delta \xi$ brings us to

$$
\delta \dot{\xi}=[D f(s(t))-2 C] \delta \xi,
$$

where $s(t)$ is the considered synchronous motion (3). Equation (4) can be obtained as the transverse part of the varia-

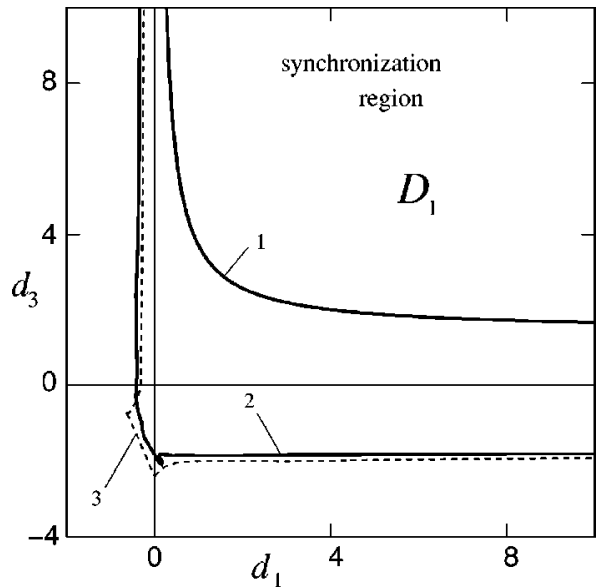

FIG. 2. Regions of synchronization obtained by different methods. Curve 1 corresponds to the Lyapunov function method; curve 2 is the blowout bifurcation curve; curve 3 delineates the transverse stability region for the equilibrium point $O$. Here $d_{2}=1$ is fixed.

tional equation for the synchronous solution (3). The longitudinal part coincides with the variational equation for the individual system $\delta \dot{\eta}=D f(s(t)) \delta \eta$. Hence, the local stability properties of the synchronous attractor $A$ are given by the stability properties of the equilibrium $\delta \xi=0$ of system (4) with $s(t) \in \mathcal{A}$.

\section{CONDITIONS FOR SYNCHRONIZATION}

Most methods for obtaining the conditions of synchronization described in the literature ${ }^{1,3,27,29,35}$ are based on an investigation of Eq. (4), i.e., they exploit local analysis. We can roughly divide them into two groups: analytical and numerical.

\section{A. Analytical methods}

Applying the Lyapunov function criteria with the function $V=\|\delta \xi\|^{2}$ to Eq. (4) we obtain the following sufficient conditions for synchronization: ${ }^{35,36}$

$$
\delta \xi^{T}\left(D f^{T}(s)+D f(s)-4 C\right) \delta \xi<0,
$$

which must be fulfilled for all points $s$ of the attractor $\mathcal{A}$ and for all nonzero $\delta \xi$. Figure 2 shows the region $D_{1}$ of synchronization as determined from Eq. (5) for our coupled Rössler systems. More precisely, ${ }^{29}$ if the coupling parameters satisfy $d_{2}>a / 2$ and $\left(d_{1}, d_{3}\right) \in D_{1}$ then the attractor $A$ [see Fig. 1(a)] of system (1) is asymptotically stable. Note that all three coupling parameters must be "large enough" to guarantee the synchronization. This observation is in agreement with the results obtained by Pecora et al., ${ }^{3}$ stating that coupled Rössler systems exhibit a desynchronization transition with increasing $d_{1}$ in the case where $d_{2}=d_{3}=0$.

Another analytical method, ${ }^{27}$ which allows us to approximate the synchronization region, is based on a calculation of the transverse stability of some simple synchronous orbit for the coupled system (1). Consider, for example, our system of coupled Rössler oscillators. It is known that, in the individual system, near the chaotic attractor there exists a saddle equilibrium point, cf. point $O$ in Fig. 1(a). We can determine the transverse stability properties for this point 
analytically ${ }^{27}$ and compare the results with other criteria for synchronization. The transverse stability region of the equilibrium point is shown in Fig. 2 as the upper-right region delineated by the dashed line. Comparing with the exact desynchronization threshold, in the next section we will show that, at least for the considered Rössler system, this method gives a reasonable approximation to the synchronization region for the chaotic attractor. The advantage of the abovementioned method is that it provides a relatively simple analytical expression that can be used for any coupling matrix $C$. For example, we have showed in Ref. 27 that for firstcomponent coupling $d_{2}=d_{3}=0$, the equilibrium undergoes a similar desynchronization transition at large $d_{1}$ as the chaotic attractor itself. Sometimes, this approach also allows us to determine whether the riddling bifurcation is sub- or supercritical. ${ }^{27}$

\section{B. Transverse Lyapunov exponents and low-periodic orbits}

The minimal condition for transverse stability of the fully synchronized state is to have the transverse Lyapunov exponents associated with Eq. (4) be negative. ${ }^{3}$ Generally speaking, these exponents depend on the orbit $s(t)$. Numerical "long-time" simulations reveal the average transverse stability of the chaotic attractor or the transverse stability of typical orbits embedded in this attractor. In the following when speaking about transverse Lyapunov exponents we understand those calculated along a typical orbit, unless other orbits are explicitly mentioned. For the case of coupled Rössler systems, the boundary of the region where the transverse Lyapunov exponent is negative is shown in Fig. 1 as curve 2. The negativeness of the transverse Lyapunov exponents ensures only the transverse stability of the chaotic attractor on "average" while some nontypical, transversely unstable orbits may still exist in the attractor. Hence, we conclude that negativeness of the transverse Lyapunov exponents gives only the necessary conditions for synchronization. To obtain the precise desynchronization threshold it is necessary to investigate the transverse stability of nontypical orbits in the attractor, e.g., saddle periodic cycles. The importance of unstable periodic orbits for the description of the properties of chaotic attractors was pointed out in Refs. 37 and 38 and, in particular for the blowout bifurcation, in Refs. 39-41. In connection with the stability properties of the fully synchronized chaotic state, unstable periodic orbits may determine the riddling bifurcation, i.e., the moment when the first orbit on the attractor loses its transverse stability. ${ }^{3,27}$ Figure 3 shows a two-dimensional stability diagram for the low-periodic orbits embedded in the chaotic attractor. Dotted and thin curves delineate regions of transverse stability for each of these orbits (up to period 4). The bold curve denotes the blowout bifurcation. The upper-right envelope of the dotted curves determines when the first of the considered periodic orbits loses its transverse stability, i.e., it approximates the riddling bifurcation. Here we have assumed that the desynchronization thresholds for high periodic orbits are closer to the blowout bifurcation and, hence, do not contribute directly to the riddling. Figure 4(a) shows the transverse stability threshold for the period-10 orbit, cf. Fig. 4(b). Note

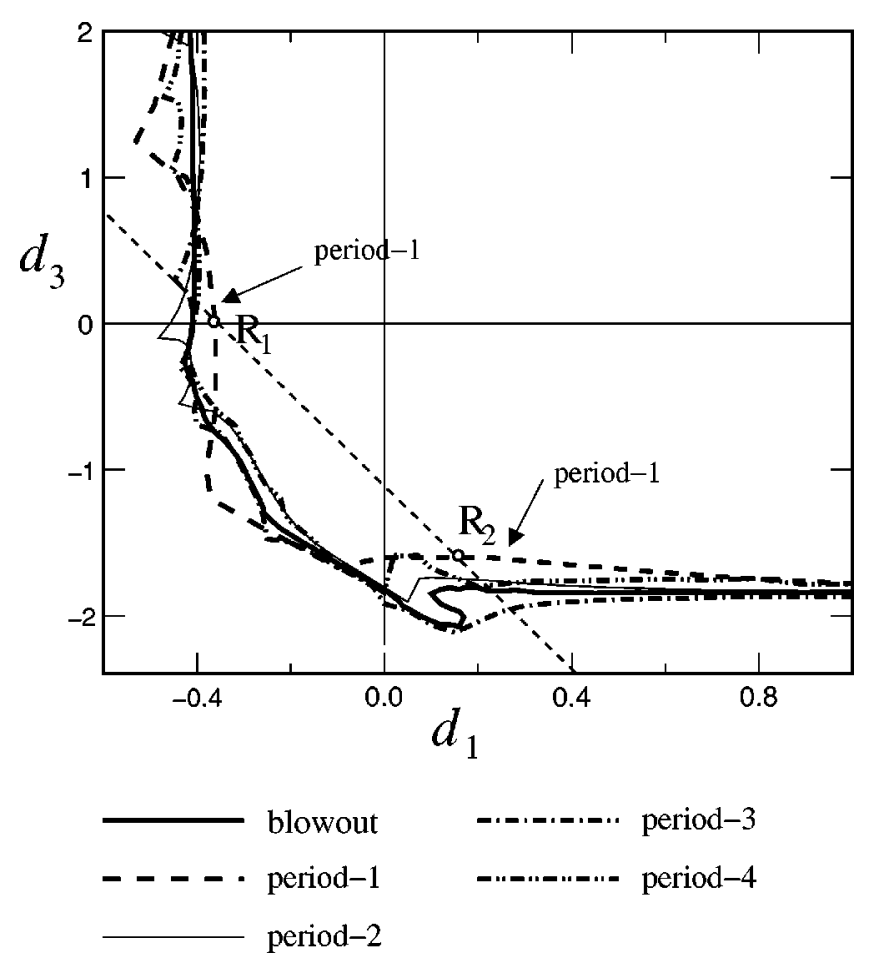

FIG. 3. Thresholds of transverse stability for low-periodic orbits (dotted and thin lines) together with the blowout bifurcation curve (heavy line).

how close this threshold is to the blowout bifurcation curve. A qualitative explanation of this fact is that high-periodic orbits for the Rössler system with the considered parameter values cover the attractor rather "uniformly" and hence, have average properties that are close to those of the chaotic attractor. Further evidence for the importance of lowperiodic orbits in the desynchronization of our system will be given in Secs. IV and V. Here, we will show how the structure of the riddled basins can be explained by exploring transverse bifurcations associated with the period-1 orbit. As previously mentioned we have performed a similar analysis for a system of two coupled pancreatic cells. ${ }^{29}$ It is interesting to note the high degree of qualitative concordance of the results between the two systems.

\section{MECHANISMS OF DESYNCHRONIZATION}

\section{A. Blowout bifurcation and desynchronization thresholds}

Blowout takes place when the transverse Lyapunov exponent of the synchronous chaotic set becomes positive and this set becomes a repeller on average. The curve where this bifurcation occurs for the coupled Rössler systems is shown in Figs. 2 and 3. The region of weak synchronization is delineated by the blowout curve. In this region it is possible to observe not only complete synchronization but also on-off intermittency ${ }^{22-24}$ caused by the existence of transversely unstable periodic orbits.

As we have seen in Sec. III, synchronization can be achieved when all three coupling parameters $d_{1}, d_{2}$, and $d_{3}$ are sufficiently large. For example, one-component coupling only, i.e., $d_{2}=d_{3}=0$, we observe a desynchronization transition in the coupled Rössler systems with increasing coupling 

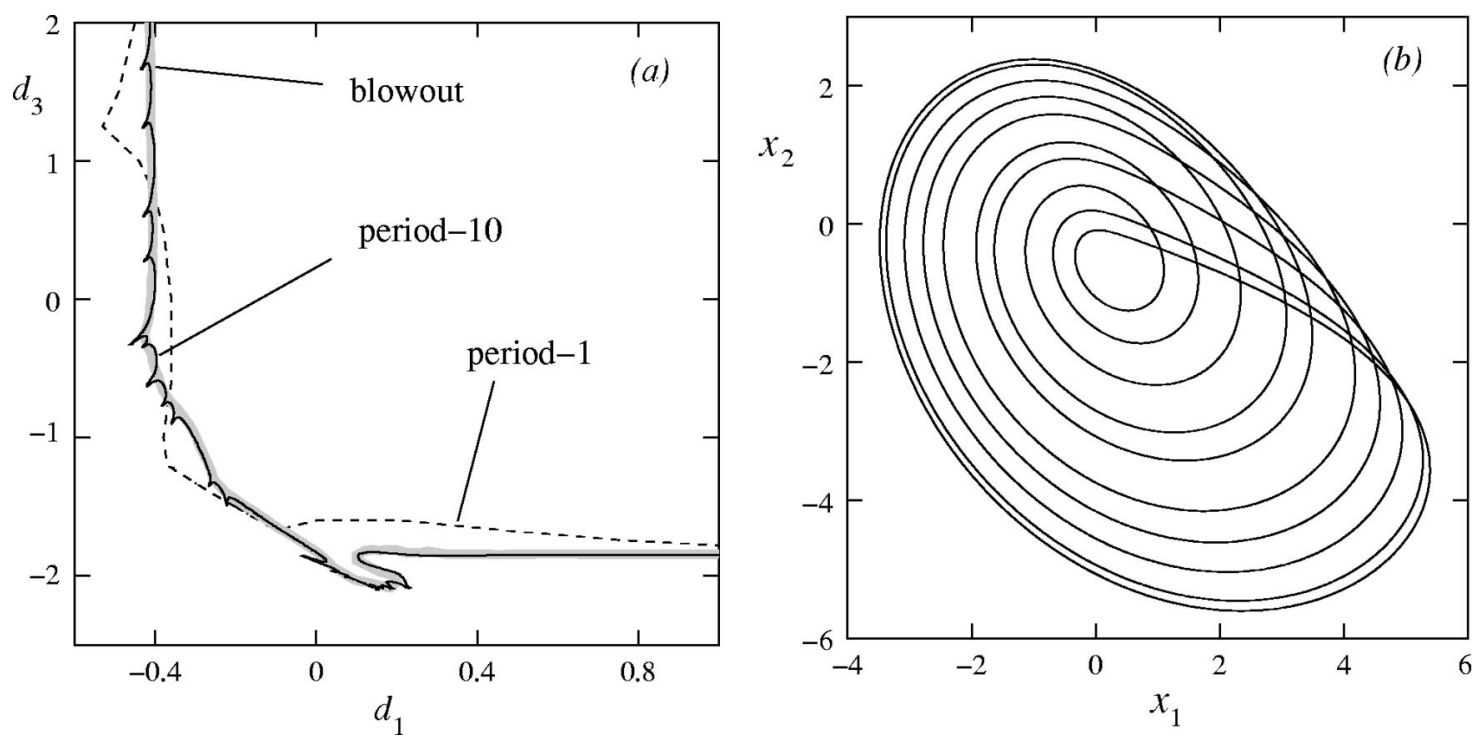

FIG. 4. Transverse stability region for the period-10 cycle (a). The boundary is shown by the solid black line. The cycle is shown in (b). The blowout curve (heavy gray line) and the boundary of the stability region for the period-1 cycle (dashed line) are shown for comparison.

parameter $d_{1} \cdot{ }^{3}$ An interesting case may also be observed when $d_{1}=0.14$ and $d_{2}=1.0$ are fixed and $d_{3}$ is increasing, cf. Fig. 5. Under these conditions we find a synchronizationdesynchronization-synchronization transition. This phenomenon arises because of the multivalued form of the blowout bifurcation curve in the bottom part of the bifurcation diagram in Fig. 4(a).

\section{B. Riddling bifurcations}

The possible riddling bifurcations in the case of two symmetrically coupled Rössler oscillators include transverse period-doubling, pitchfork, and Neimark-Sacker bifurcations of cycles of periods 1,2 , and $3^{27}$ (the Neimark-Sacker bifurcation is also referred to as a secondary Hopf bifurcation or a torus-birth bifurcation). Inspection of Fig. 3 shows that a large part of the riddling bifurcation curve is associated with the loss of transverse stability for the period-1 cycle.

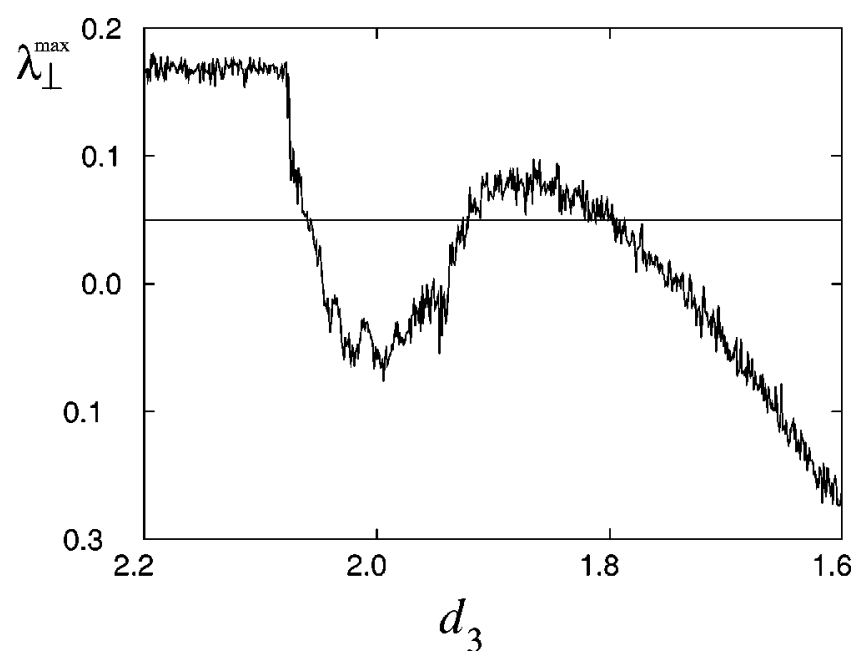

FIG. 5. Nontrivial behavior of the largest transverse Lyapunov exponent as a function of $d_{3} \cdot d_{1}=0.14$ and $d_{2}=1.0$.
For the purpose of illustration, we confine our attention to parameter values where the period-1 cycle plays the most important role. With this aim we introduce a new coupling parameter $d$ such that its variation corresponds to a scan along the line $R_{1} R_{2}$ in Fig. 3. In terms of this new parameter, the coupling matrix becomes

$$
C=\operatorname{diag}\{d-0.6,1.0,-3.1 d+0.7\} .
$$

The line $R_{1} R_{2}$ was chosen such that the region of complete synchronization in the middle of the scan is bounded by the points $R_{1}$ and $R_{2}$, which both correspond to riddling bifurcations for the period-1 cycle. Point $R_{1}(d=0.2408)$ corresponds to a supercritical period-doubling bifurcation, and $R_{2}$ ( $d=0.7360)$ to a subcritical pitchfork bifurcation. Let us now investigate the associated bifurcations by application of the one-dimensional continuation technique. As we will show, the nature of these bifurcations play an important role for the global dynamics of the system. Figure 6 provides a schematic overview of the bifurcation diagram versus param-

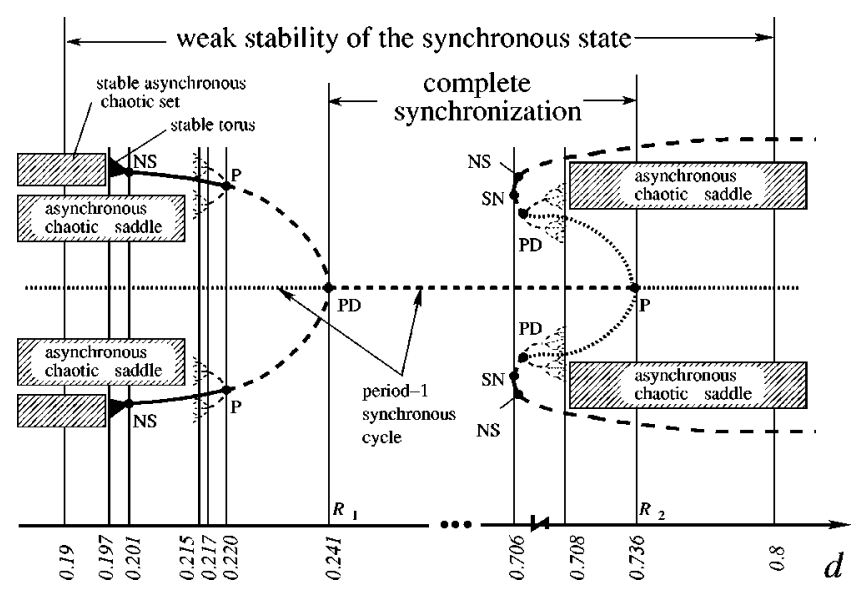

FIG. 6. Bifurcation diagram for the period-1 cycle and its associated solutions vs the coupling parameter $d$. Points $R_{1}$ and $R_{2}$ denote moments when the transverse stability of the synchronized period-1 cycle is changed. 

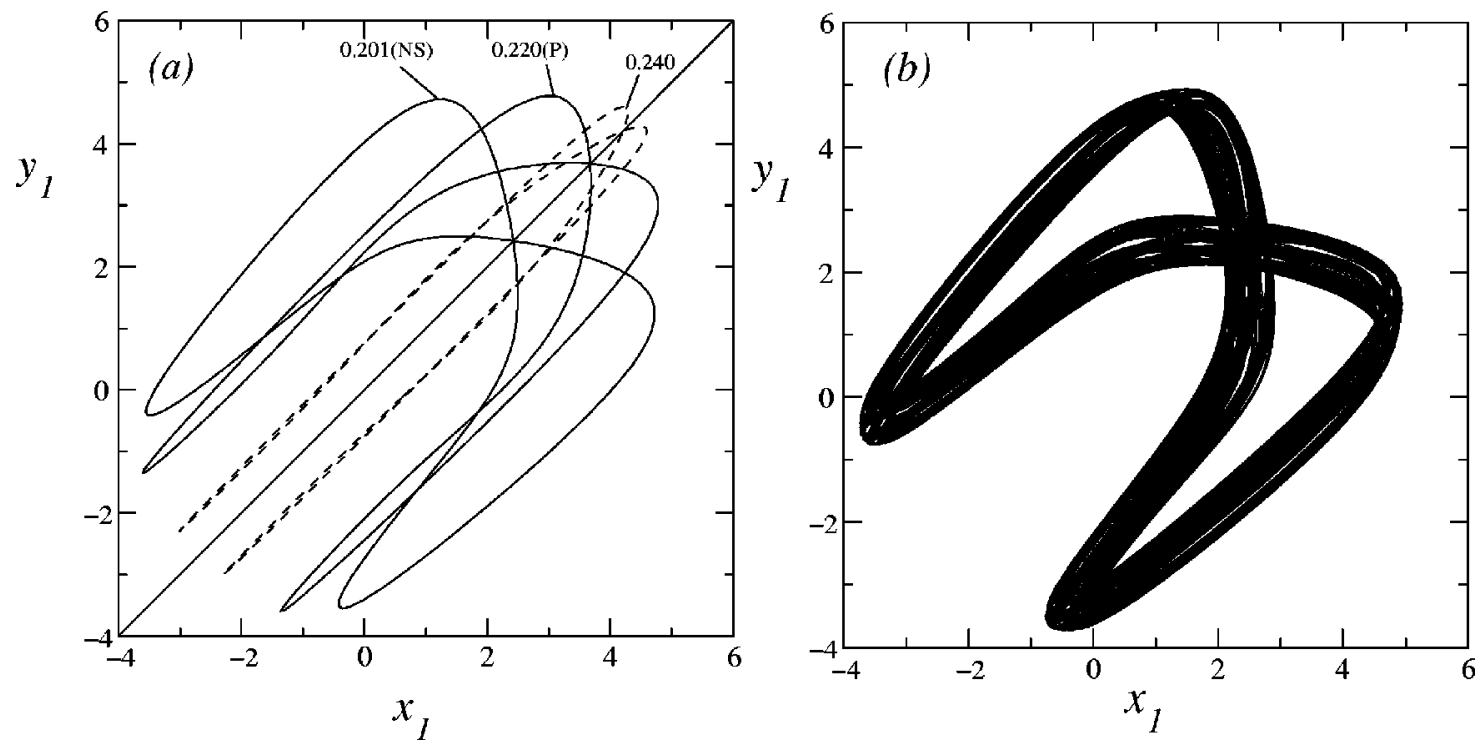

FIG. 7. (a) Evolution of the period- 2 asynchronous cycle in phase space for the parameter interval $0.201<d<0.241$. A stable cycle is shown as a solid line. (b) Stable asynchronous torus at $d=0.2$. Projection onto the $\left(x_{1}, y_{1}\right)$ plane.

eter $d$ for the period-1 cycle and the associated asynchronous solutions. The horizontal line in the middle of Fig. 6 corresponds to the synchronous period-1 cycle. This line is dashed for parameter values where the cycle is transversely stable and has a single unstable longitudinal multiplier, i.e., in the parameter interval between points $R_{1}$ and $R_{2}$. The dotted part of the line indicates parameter values for which the synchronized period-1 cycle is transversely unstable. At $d$ $=0.241$ (point $R_{1}$ ) the cycle undergoes a transverse supercritical period-doubling bifurcation, cf. point $P D$, and an asynchronous period-2 cycle appears. When decreasing the parameter $d$, this period-2 cycle becomes stable at point $P$ $(d=0.220)$. The evolution of this cycle in phase space is shown in Fig. 7(a). A Neimark-Sacker bifurcation takes place at $d=0.201$ (point $H$ ) and gives rise to a stable asynchronous torus, cf. Fig. 7(b), which is destroyed in a transition to chaos at $d=0.197$. After the torus breakdown, iterations of the Poincaré map produce the asynchronous chaotic set displayed in Fig. 8. Similarly, the right part of the bifurcation diagram in Fig. 6 displays bifurcations that occur with increasing parameter $d: P$ is a subcritical pitchfork bifurcation (point $R_{2}$ ), which leads to the appearance of two mutually symmetric, doubly unstable period- 1 cycles outside the synchronization manifold.

Important observations to be made from the bifurcation diagram in Fig. 6 are the following:

(1) the region of complete synchronization of the synchronous chaotic set is $0.241<d<0.736$;

(2) the region where the synchronous set is transversely stable on average (i.e., weakly stable) is approximately $0.19<d<0.80$

(3) there exists an asynchronous chaotic saddle even for parameter values of complete synchronization $(0.708<d$ $<0.736$ );

(4) there are parameter regions with coexistence of the weakly stable synchronous chaotic set with a stable asynchronous cycle $(0.201<d<0.220)$, a stable asynchronous torus $(0.197<d<0.201)$, and a stable asynchronous chaotic set $(d<0.197)$;

(5) when increasing the parameter $d$ to the right-hand side of $R_{2}$, no stable asynchronous sets are detected.

In Sec. V, we will make use of the above-mentioned information to explain the structure of the riddled basins that can be observed in our coupled Rössler system.

\section{INFLUENCE OF RIDDLING BIFURCATIONS ON RIDDLED BASIN STRUCTURES}

Riddled basins of attraction ${ }^{20,22}$ may be observed after the riddling bifurcation as long as the maximal transverse Lyapunov exponent is still negative for typical trajectories.

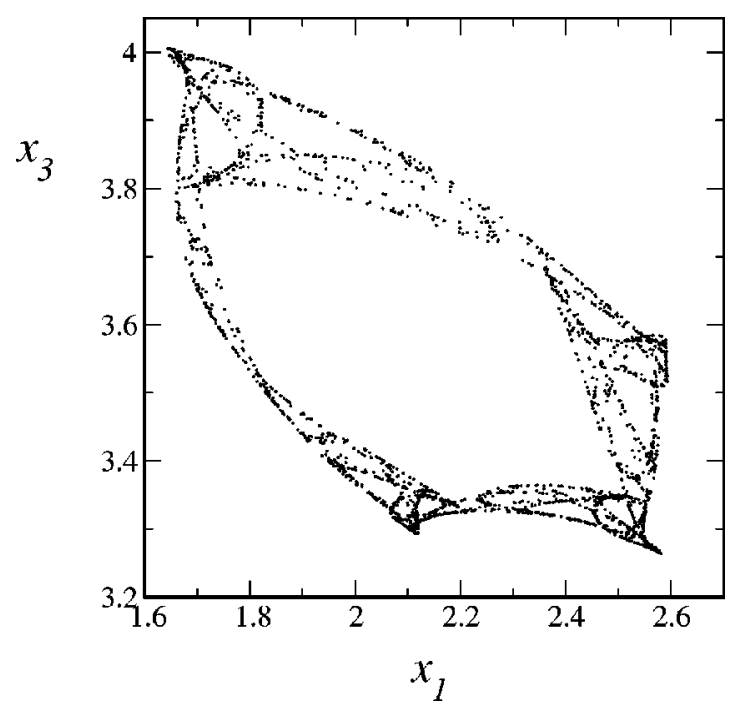

FIG. 8. Projection onto the $\left(x_{1}, x_{3}\right)$ plane of the invariant set of the Poincare map for $d=0.197$. 


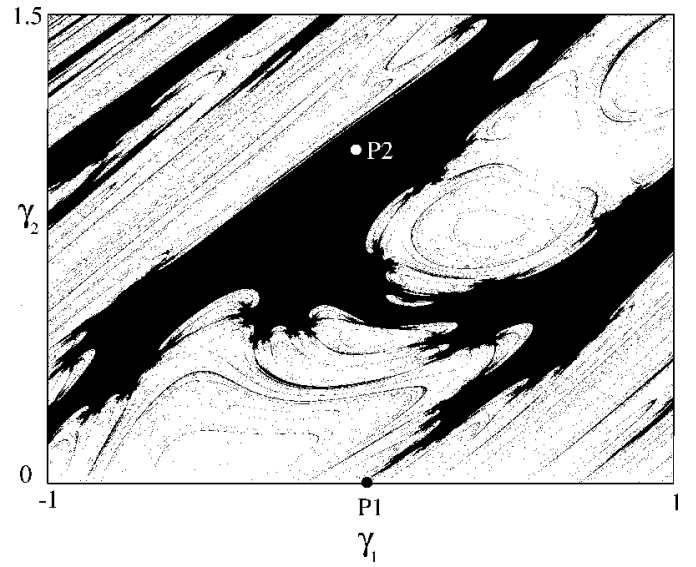

FIG. 9. Riddled basin of attraction for the synchronous chaotic set (white points) with $d=0.204$. Black points represent initial conditions from which the trajectory is attracted to the stable period- 2 asynchronous cycle. $P_{2}$ is the point of intersection with the period-2 cycle and $P_{1}$ with the synchronous period-1 cycle. $\gamma_{1}$ and $\gamma_{2}$ are the coordinates on the considered plane such that $\gamma_{2}=0$ corresponds to the synchronization manifold.

Hence, the parameter region where riddling may occur is bounded by the blowout bifurcation from one side and by the riddling bifurcation from the other. With respect to the coupling parameter $d$, this region consists of two pieces: 0.19 $<d<0.241$ and $0.736<d<0.8$, cf. Fig. 6. It follows from Fig. 6 that the basin of the synchronous chaotic set is riddled with points belonging to the basin of a stable asynchronous period-2 cycle for parameter values in the interval 0.201 $<d<0.220$. Similarly, the basin will be riddled with points attracted to an asynchronous torus for $0.197<d<0.201$ and to a stable asynchronous chaotic set for $0.19<d<0.197$. For other parameter values where riddling occurs we may observe either attractor bubbling or riddling with points diverging to infinity. The first situation generally arises when the riddling bifurcation is supercritical. ${ }^{42}$ Therefore, riddling with infinity is expected for the parameters $0.736<d<0.8$. Further evidence for this will be given in Sec. VIII, where we consider the problem of boundedness of the solutions of the coupled system.

In view of the above-mentioned considerations, we calculated two-dimensional cross sections of the basins of attraction for different values of $d$. Figure 9 corresponds to $d$ $=0.204$. Black points denote initial conditions that converge to the asynchronous period-2 cycle and white represents points converging to the weakly stable synchronous chaotic set. Figure 10 shows how the basin of attraction for the synchronized chaotic state (white points) is riddled with points (black) from which the trajectory diverges to infinity. Here, $d=0.75$.

Some of the tongues of the riddled basins are clearly seen in Figs. 9 and 10, e.g., the black region emanating from the point $P 1$. The probability to diverge from the synchronization manifold is highest when the initial conditions are chosen in the neighborhood of these points. In both cases the points $P 1$ belong to the period- 1 synchronous cycle embedded in the chaotic set, i.e., the cycle which loses its transverse stability first. Hence, the neighborhood of this cycle is

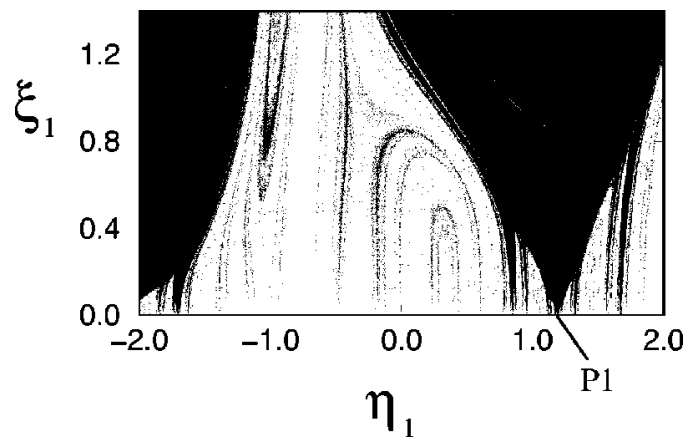

FIG. 10. Riddled basin of attraction for the synchronous chaotic set (white points) with $d=0.75$. Black points correspond to initial conditions diverging to infinity.

most exposed to escapes from the weakly stable synchronization manifold.

In order to identify the location of the other tongues of the riddled basin, we have investigated the long-term behavior of trajectories starting from the following set of points: $\eta \in \mathcal{A}, \quad \xi=(0.01,0,0)$, i.e., with longitudinal coordinates at the synchronous set and a small transverse perturbation. The projection of this set onto $\left(x_{1}, x_{2}\right)$ plane has a form similar to the Rössler attractor. For a fixed $d=0.75$ (this corresponds to Fig. 10), we can distinguish two types of points: points that eventually converge to the synchronization manifold and points that diverge to infinity. Points diverging to infinity are marked black in Fig. 11(a). Inspection of the figure shows that the densest parts are located near the synchronous period-1 cycle. In order to explain the remaining structure of the basin of attraction, we have used the Poincare section $S$, cf. Fig. 1, of the individual Rössler oscillator. Figure 12(a) shows the invariant attracting set of this map. Considering this set to be nearly one-dimensional, we have constructed the one-dimensional map $h: x_{3} \rightarrow h\left(x_{3}\right)$ acting on the set, cf. Fig. 12(b). The fixed point $P$ of this map corresponds to the transversely unstable period- 1 cycle. We will consider the orbits that start from the different preimages of $P: P^{-1}$, $P^{-2}, P_{1,2}^{-3}, P_{1,2,3,4}^{-4}$, and so on. These orbits approach the period-1 cycle in one, two, three, and four loops, respectively. In this way, considering different preimages of the fixed point $P$, we may find different orbits of the Rössler system which after a number of loops enter the neighborhood
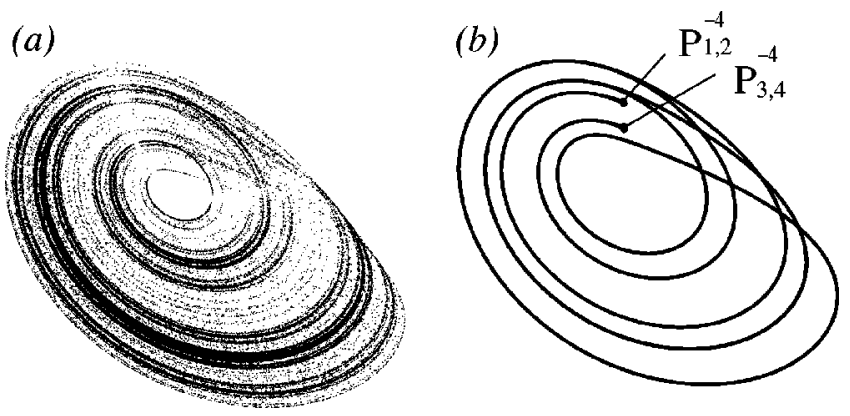

FIG. 11. (a) Given a point on the synchronous attractor, we add a small fixed transverse perturbation $\xi=(0.01,0,0)$. For the obtained initial condition, the point is marked black if the orbit diverges to infinity. (b) Orbits starting from $P_{1,2,3,4}^{-4}$ 
(a)

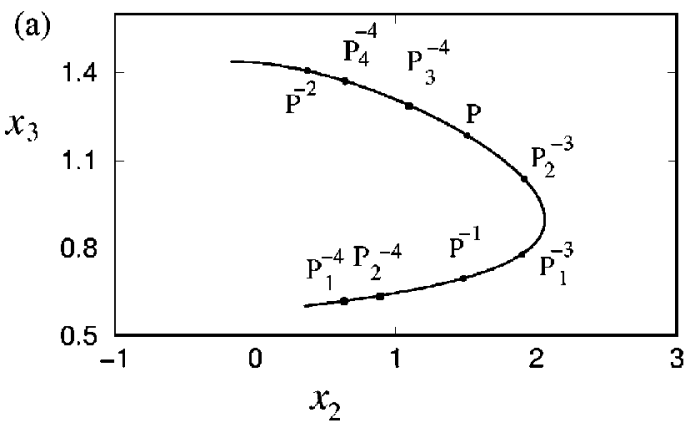

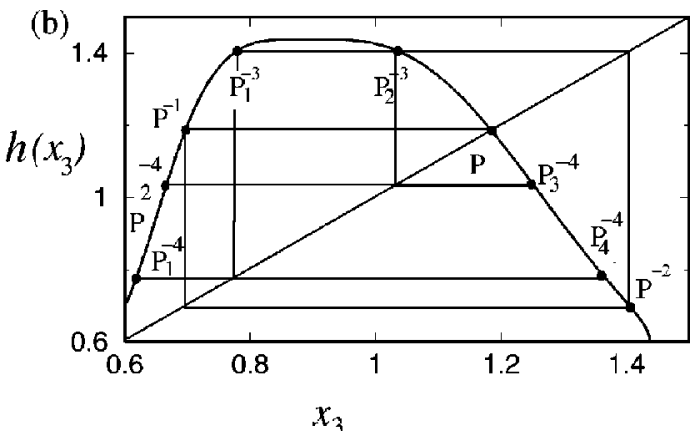

FIG. 12. Invariant set of the Poincaré map for the individual Rössler oscillator (a). One-dimensional map acting on the invariant set (b).

of the period-1 cycle and, hence, share the property of transverse instability with the period-1 cycle. These orbits form the skeleton of the riddling structure in Fig. 11(a). The orbits starting from $P_{1,2,3,4}^{-4}$ are shown in Fig. 11(b). We observe that these orbits correspond to the transversely most unstable parts of the synchronized attractor.

To conclude this section, we note that the transverse stability properties of the low-periodic orbits embedded in the synchronized chaotic state are very important for explaining the onset of riddling and describing the structure of the riddled basin for coupled systems.

\section{NONIDENTICAL SYSTEMS}

\section{A. "Shift" of the synchronization manifold for strong coupling}

One of the most important questions in connection with the application of chaotic synchronization for secure communication and other purposes relates to the sensitivity of the synchronized state to a mismatch in the parameter values between the interacting systems. Some general estimates of the influence of a parameter mismatch were obtained by Johnson et al. ${ }^{43}$ Using an approach similar to theirs, we have obtained asymptotic estimates of the solutions for a special type of parameter mismatch. ${ }^{29}$

Let us assume a special form of the mismatch, namely, that the coupled system can be written as

$$
\begin{aligned}
& \dot{x}=f(x)+\varepsilon g+C(y-x), \\
& \dot{y}=f(y)+C(x-y),
\end{aligned}
$$

where $\varepsilon$ is a parameter that measures the magnitude of the mismatch and $g$ is a constant vector. $f$ is a smooth function. Despite its special form, Eq. (7) has a wide scope of applications. Examples are:

(1) two coupled systems with a mismatch of parameters that are included in an additive way (e.g., two coupled Rössler systems with different parameters $b$ );

(2) two coupled systems of the form (1) with a constant external force acting on one of the oscillators (e.g., two coupled $\beta$ cells with an additional constant leak current $^{29}$ );

(3) two coupled systems with external forces where the frequency of the external force is much smaller than the natural frequency of the individual oscillator.
We have shown ${ }^{29}$ that for the vector coupling $C=\alpha I$, where $I$ is the identical matrix, the following asymptotic estimation holds:

$$
\left|\xi(t)-\frac{\varepsilon g}{2 \alpha}\right| \leqslant L \frac{l \varepsilon g}{\alpha^{2}} .
$$

The inequality and the modulus $|\cdot|$ should be considered componentwise. $L$ is some constant that does not depend on the coupling or mismatch. $l$ is a constant that expresses the smoothness of the system, namely, $\|D f(s(t))\| \leqslant l$ for the motion on the attractor. Finally, as before, $\xi$ is the transverse coordinate measuring the distance from the synchronization manifold. To derive Eq. (8), we assumed the mismatch to be small $\varepsilon \ll l$ and $\varepsilon \ll \alpha$. Moreover, we supposed that the coupling is sufficiently strong to guarantee complete synchronization in the system without mismatch.

As a consequence of the obtained inequality, the influence of a parameter mismatch can be regarded as the superposition of the following effects.

(1) Displacement of the synchronous motion from the synchronization manifold by an amount $\varepsilon g / 2 \alpha$. The direction of this shift depends on the sign of the parameter mismatch.

(2) Chaotic bursts around the shifted state with amplitudes of the order of $1 / \alpha^{2}$. For large coupling strengths $\alpha$, the bursts become small compared to the shift, and one can numerically observe the phenomenon of generalized synchronization where $\|x(t)-y(t)\| \rightarrow$ const $\neq 0$.

Despite the fact that the inequality (8) was proved for a vector coupling, numerical simulations show that qualitatively the same effects take place for other coupling matrices $C$.

Figure 13 illustrates the obtained result for the case of two coupled Rössler systems. Three orbits are plotted with the coupling parameters $\alpha=2,6$, and 12, respectively. The presence of the shift away from the $y_{1}=x_{1}$ is clearly observed. We can also observe how the bursts around the shifted state are reduced with increasing of $\alpha$. The shift decreases linearly with $1 / \alpha$ while the reduction of bursts varies as $1 / \alpha^{2}$. Figure 14 shows the orbit for $\alpha=2$ in the phase space as a projection onto $\left(x_{1}, y_{1}\right)$ plane. We observe how the motion takes place at some distance from the diagonal, which represents the projection of the synchronization manifold. The nonzero width of the shifted state is caused by the 


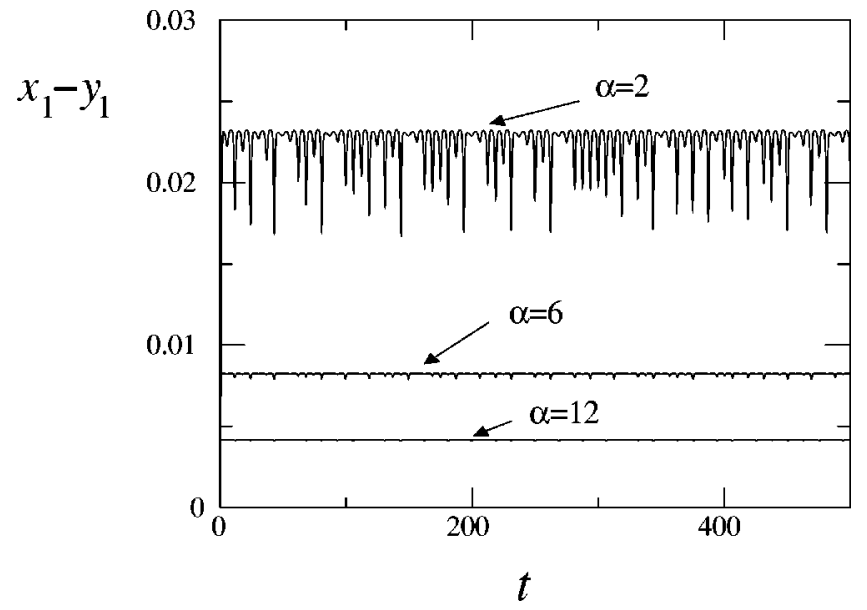

FIG. 13. Behavior of the first component of the transverse coordinate for the parameter mismatch $\varepsilon g=(0.1,0,0)^{T}$ with different values of the coupling parameter $\alpha$.

bursts. Again, it is of interest to note that qualitatively the same phenomena are observed for a system of two coupled pancreatic cells. ${ }^{29}$

\section{B. Influence of asymmetries on riddling bifurcations}

In the previous section we considered the influence of a parameter mismatch for the case of strong coupling and full synchronization. As the coupling is reduced, the synchronized state becomes weakly stable, and it is of interest to consider the effect of a mismatch on the riddling bifurcations. Here two cases may be distinguished, depending on whether the synchronization manifold still persists in the presence of the parameter mismatch and whether it no longer exists. The first case may be realized in the following asymmetrically coupled system:

$$
\begin{aligned}
& \dot{x}=f(x)+p C(d)(y-x), \\
& \dot{y}=f(y)+(2-p) C(d)(x-y),
\end{aligned}
$$

where the parameter $p$ describes the asymmetry (provided that $p \neq 1$ ). This type of coupling is of interest, for instance, when studying the transition from one- to two-cluster dy-

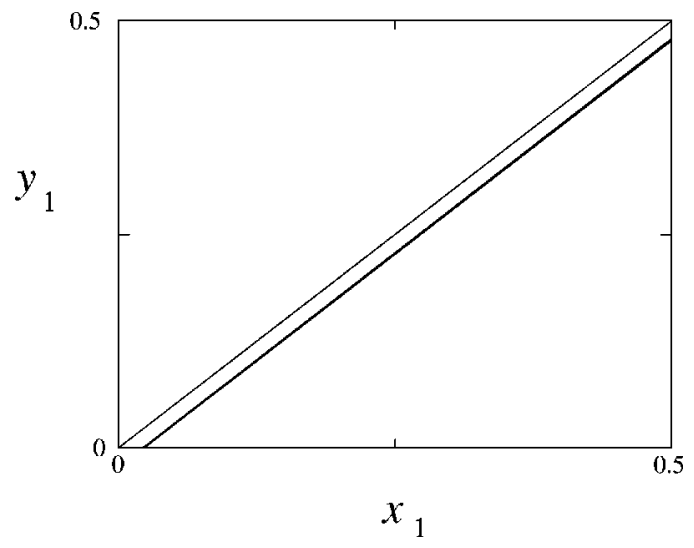

FIG. 14. Projection of the orbit onto the $\left(x_{1}, y_{1}\right)$ plane for $\varepsilon g=(0.1,0,0)^{T}$ and $\alpha=2$. Diagonal represents the projection of the synchronization manifold.

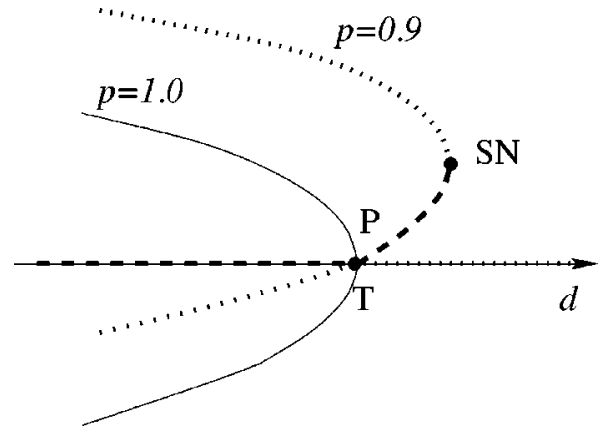

FIG. 15. Perturbation of the subcritical transverse pitchfork bifurcation for nonsymmetric coupling.

namics in ensembles of globally coupled identical chaotic oscillators. ${ }^{44}$ Considering the matrix $C(d)$ as in Eq. (6), our calculations show that the subcritical pitchfork bifurcation (occurring at $d=0.736$, cf. Fig. 6) transforms into a transcritical bifurcation. Figure 15 shows schematically the perturbed and original bifurcations. The associated saddle-node bifurcation occurs at $d \simeq 0.7382$. In the perturbed system, a doubly unstable period-1 cycle approaches the synchronization manifold and exchanges stability with the synchronous period-1 saddle cycle at the point $T$.

In the general case of a mismatch between the subsystems, the description admits the following form:

$$
\begin{aligned}
& \dot{x}=f_{1}(x)+C(d)(y-x), \\
& \dot{y}=f_{2}(y)+C(d)(x-y),
\end{aligned}
$$

where the functions $f_{1}(x)$ and $f_{2}(x)$ now are different. In this case the synchronization manifold no longer exists. The transverse pitchfork bifurcation is transformed into two isolated cycle branches, one of which undergoes saddle-node bifurcations. Figure 16 illustrates this situation for the coupled Rössler system, where $f_{1}=f(x, b)$ as in Eq. (2) with parameters $a=0.42, b=2.0, c=4.0$ and $f_{2}\left(x, b_{1}\right)$ is the same right-hand side but with $b_{1}=2.002$. Then the values of coupling parameter corresponding to bifurcations in Fig. 16: $d_{S N}=0.7286, d^{\prime}=0.7081$, and $d^{\prime \prime}=0.7056$.

\section{ARRAYS OF COUPLED OSCILLATORS: A SIMPLE EXAMPLE}

Many aspects of the above-mentioned approach may also be applied to investigate arrays of coupled identical os-

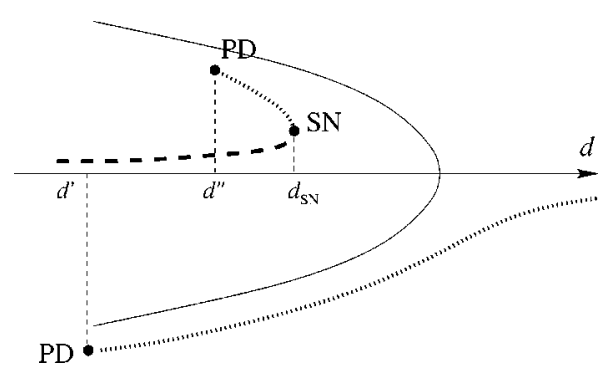

FIG. 16. Perturbation of the subcritical transverse pitchfork bifurcation in the case of nonidentical oscillators. 


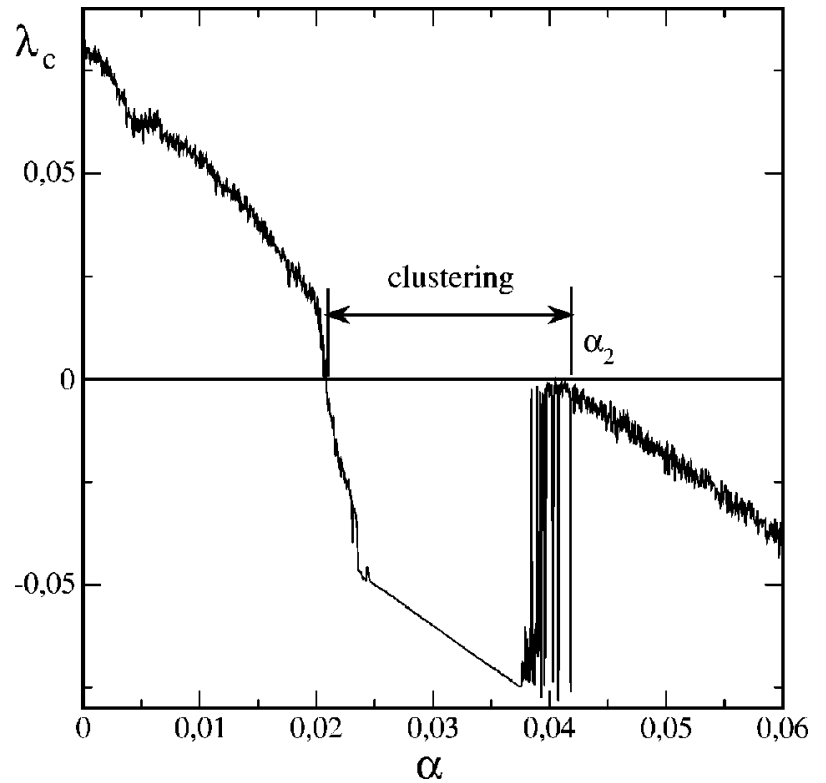

FIG. 17. Maximal Lyapunov exponent that determines the stability of the partially synchronized motion $x_{1}=x_{2}$ and $x_{3}=x_{4}$ for system (11).

cillators. Here we consider the case of four diffusively coupled Rössler oscillators with periodic boundary conditions:

$$
\begin{aligned}
& \dot{x}_{j}=f\left(x_{j}\right)+C\left(x_{j+1}+x_{j-1}-2 x_{j}\right), \quad j=1,2,3,4 ; \\
& x_{5}=x_{1}, \quad x_{0}=x_{4} .
\end{aligned}
$$

An important property of arrays of coupled oscillators is that such systems usually have many invariant hyperplanes in phase space. ${ }^{45}$ Recall, that system (1) generally has only one invariant hyperplane $x=y$ (the synchronization manifold), the stability of which determines the onset of complete synchronization. In the case of many coupled systems, the stability of different invariant hyperplanes determines the onset of partial synchronization or clustering, where the coupled system splits into clusters of identically oscillating elements.

First, it is easy to check by substitution that the following equalities are admissible for system (11):

$$
\begin{aligned}
& \Pi_{0}: x_{1} \equiv x_{2} \equiv x_{3} \equiv x_{4} ; \\
& \Pi_{1}: \quad x_{1} \equiv x_{2}, \quad x_{3} \equiv x_{4} ; \\
& \Pi_{2}: \quad x_{1} \equiv x_{4}, \quad x_{2} \equiv x_{3} ; \\
& \Pi_{3}: \quad x_{1} \equiv x_{3}, \quad x_{2} \equiv x_{4} .
\end{aligned}
$$

Hence, the hyperplanes determined by these equalities are invariant with respect to the dynamics (11). Note that the properties of the hyperplanes $\Pi_{1}$ and $\Pi_{2}$ are the same because of the symmetry of the system. As in the case of two coupled systems, the transverse stability of some invariant set located in the hyperplane $\Pi_{0}$ guarantees the complete synchronization $\left(\left\|x_{i}-x_{j}\right\| \rightarrow 0\right.$ with $t \rightarrow \infty$ for $i \neq j$ and some open set of initial conditions). Similarly, the transverse stability of a set located in $\Pi_{1}$ implies the partial synchronization when $\left\|x_{1}-x_{2}\right\| \rightarrow 0$ and $\left\|x_{3}-x_{4}\right\| \rightarrow 0$ with $t \rightarrow \infty$ for some open set of initial conditions. Hence, in order to obtain conditions for the onset of partial synchronization it is necessary to investigate the stability of invariant sets in the hyperplanes $\Pi_{1}, \Pi_{2}$, and $\Pi_{3}$. As in the case of two coupled oscillators we may consider separately longitudinal directions along the hyperplanes and directions transverse to the hyperplanes.

Consider the hyperplane $\Pi_{1}$. In order to obtain equations for the dynamics inside the hyperplane, we substitute $x_{1}=x_{2}=X$ and $x_{3}=x_{4}=Y$ into Eq. (11). We obtain two coupled systems of the form (1). It is clear that equations for the dynamics inside the hyperplane describe the endogenous motions of the clusters, i.e., the sets of identically oscillating elements of the array. Transverse coordinates may be introduced in the following way: $\xi_{1}=x_{1}-x_{2}$ and $\xi_{2}=x_{3}-x_{4}$. Similar to the derivation of Eq. (4) we may obtain the following linearized equations for the transverse perturbations to the hyperplane $\Pi_{1}$ :

$$
\delta \dot{\xi}_{1}=[D f(X(t))-3 C] \delta \xi_{1}-C \delta \xi_{2},
$$

$$
\delta \dot{\xi}_{2}=[D f(Y(t))-3 C] \delta \xi_{2}-C \delta \xi_{1},
$$

where $X(t)$ and $Y(t)$ are solutions of the system of two coupled oscillators (1). Observe that we now have six transverse and six longitudinal coordinates.

As an illustrative example, let us consider coupled Rössler systems with vector coupling $C=\alpha I$. Figure 17 shows the largest transverse Lyapunov exponent [i.e., the Lyapunov exponent for system (12)] versus the coupling parameter $\alpha$. It can be seen that after the loss of complete synchronization at $\alpha_{2}=0.04$ we have a wide range $0.02<\alpha$ $<0.04$ where the Lyapunov exponent is negative. This corresponds to a stable, partially synchronous state with $x_{1}$ $=x_{2}$ and $x_{3}=x_{4}$. Inspection of the longitudinal dynamics ${ }^{28}$ shows that for these parameters the hyperplane contains a stable periodic solution. Hence, we have shown that for $0.02<\alpha<0.04$ there exists a stable limit cycle in the hyperplane $\Pi_{1}$ which implies the existence of periodic clusters with $x_{1}=x_{2}$ and $x_{3}=x_{4}$. Taking the symmetry into account, we conclude that there is also a stable limit cycle inside the hyperplane $\Pi_{2}$, which implies the existence of periodic clusters with $x_{1}=x_{4}$ and $x_{2}=x_{3}$. Figure 18 illustrates both clusters for $\alpha=0.035$. With different initial conditions we may approach either of these two clusters with equal probability. It appears that in the considered case the basins of attraction of the two attracting cycles are strongly mixed. This can be seen on the two-dimensional cross section of the basins in Fig. 19.

We have seen that partial synchronization in arrays of coupled oscillators is mediated by the existence of stable invariant sets in the invariant hyperplanes (or manifolds). The stability of these sets may be investigated by a decomposition into longitudinal and transverse coordinates as in the case of two coupled oscillators. 
(a)
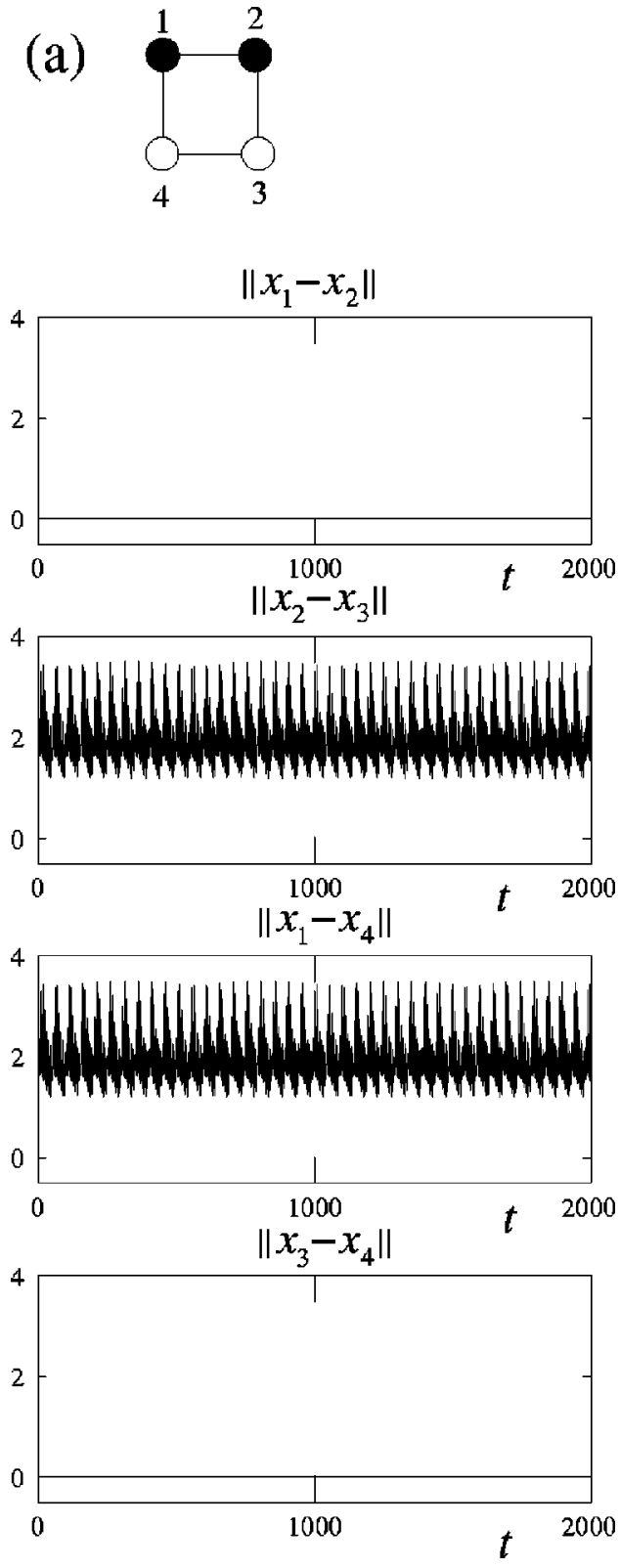

(b)

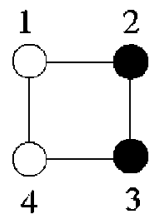

$\left\|x_{1}-x_{2}\right\|$
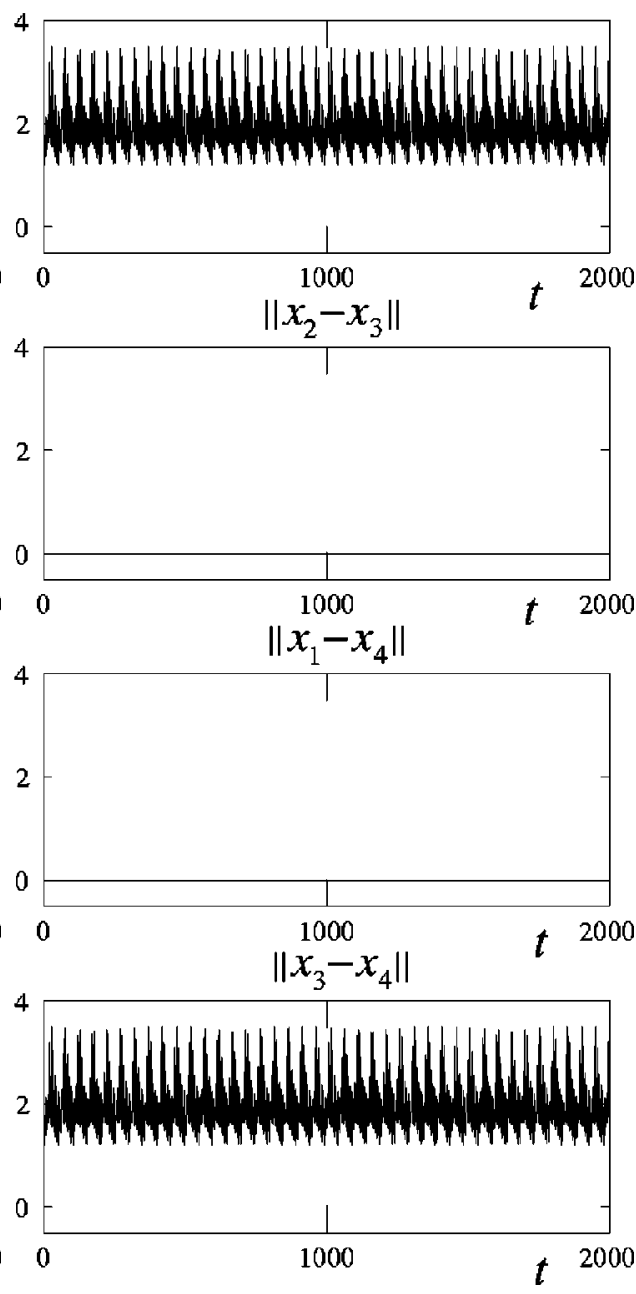

FIG. 18. Two orbits that correspond to two clusters that can be realized in system (11) for $\alpha=0.035$. (a) $x_{1}=x_{2}$ and $x_{3}=x_{4}$ for one set of initial conditions; (b) $x_{1}=x_{4}$ and $x_{2}=x_{3}$ for another set of initial conditions.

\section{CONCLUSION}

Let us conclude the paper with a discussion of the boundedness of the solutions to a system of two coupled, time-continuous oscillators. As discussed in Sec. V, for some parameter values there exist orbits in the vicinity of the synchronization manifold that diverge to infinity, while for other parameters the orbits are confined to some bounded domain in the phase space. Moreover these two cases can be observed even for a coupling strength strong enough to guarantee weak synchronization.

In this section we present a general result that provides conditions for the existence of a positively invariant region around the synchronized attractor. ${ }^{46} \mathrm{~A}$ positively invariant region (or trapping zone) is defined by the condition that trajectories that enter the region will never leave it again.

Suppose system (1) satisfies the following conditions:

(1) The coupling matrix is of the form $C=\alpha I$.
(2) For an individual oscillator equation $\dot{x}=f(x), x \in R^{n}$ there exists an invariant attracting set $\mathcal{A}$ and $U_{\mathcal{A}}$ is the region of attraction for this set. (Instead of this condition we may simply suppose the existence of positively invariant set $U_{\mathcal{A}}$-Ref. 46.)

(3) $U_{\mathcal{A}}$ is convex.

Then for any $\alpha>0$ system (1) will have a positively invariant set $U_{\mathcal{A}} \times U_{\mathcal{A}}$ around the synchronized attractor $A$ in the phase space $R^{2 n}$.

A strict mathematical formulation of the above-given theorem was given in Ref. 46. There we also supposed a good local structure of the set $U_{\mathcal{A}}$. Note that the existence of the positively invariant region is proved for values of the coupling parameter corresponding not only to complete synchronization but also to weak synchronization and to the desynchronized regime. This brings us to the conclusion that the vector coupling scheme, in general, does not exhibit riddling with infinity. Moreover, in view of this result, riddling 


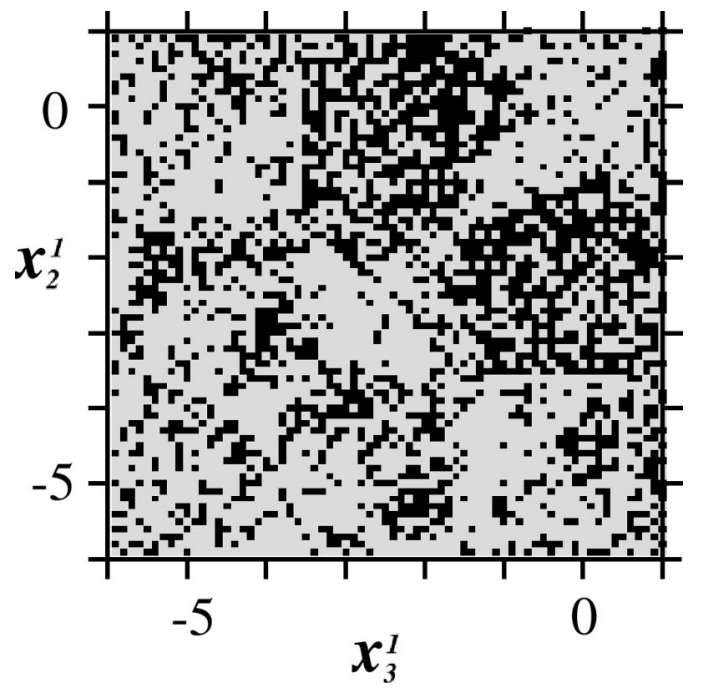

FIG. 19. Two-dimensional cross section of the phase space determined by $x_{1 i}=1.0, x_{4 i}=1.0, x_{2 j}=1.0$, and $x_{3 j}=1.0$, where $i=1,2,3,4$ and $j=1,2,3$. A $70 \times 70$ grid is used. A square is painted black if the orbit with initial conditions from the center of the square converges to the cycle corresponding to the $x_{1}=x_{2}, x_{3}=x_{4}$ cluster.

with infinity may be expected when the coupling matrix $C$ differs significantly from identical. These observations are also supported by our calculations in Sec. V.

Let us finally conclude that the system of two coupled oscillators is important for studying many nonlinear phenomena. The importance of studying objects with invariant hyperplanes is clear not only from the point of view of complete identical synchronization of two coupled systems. This is also one of the widely accepted approaches for studying partial synchronization in arrays of coupled oscillators.

\section{ACKNOWLEDGMENTS}

S.Y. acknowledges support by INTAS (Grant No. YSF 01-084), SFFI of Ukraine (Grant No. 01.07/00109), and by the Danish Graduate School in Nonlinear Science.

${ }^{1}$ H. Fujisaka and T. Yamada, "Stability theory of synchronized motion in coupled oscillator systems," Prog. Theor. Phys. 69, 32-46 (1983).

${ }^{2}$ N. F. Rul'kov, "Images of synchronized chaos: Experiments with circuits," Chaos 6, 262-279 (1996).

${ }^{3}$ L. M. Pecora, T. L. Carroll, G. A. Johnson, D. J. Mar, and J. F. Heagy, "Fundamentals of synchronization in chaotic systems, concepts, and applications," Chaos 7, 520-543 (1997).

${ }^{4}$ L. M. Pecora and T. L. Carroll, "Synchronizing chaotic systems," IEEE Trans. Circuits Syst., I: Fundam. Theory Appl. CS-38, 453-456 (1996).

${ }^{5}$ M. Sushchik, N. Rulkov, and L. Larson, "Chaotic pulse position modulation: A robust method of communication with chaos," IEEE Comm. Lett. 4, 128-130 (2000).

${ }^{6}$ R. Roy and K. S. Thornburg, "Experimental synchronization of chaotic lasers," Phys. Rev. Lett. 72, 2009-2012 (1994).

${ }^{7} \mathrm{~K}$. Wiesenfeld and P. Hadley, "Attractor crowding in oscillator arrays," Phys. Rev. Lett. 62, 1335-1338 (1989).

${ }^{8}$ W. Wang, I. Z. Kiss, and J. L. Hudson, "Experiments on arrays of globally coupled electrochemical oscillators: Synchronization and clustering," Chaos 10, 248-256 (2000).

${ }^{9}$ H. Bohr, K. S. Jensen, T. Petersen, B. Rathjen, E. Mosekilde, and N.-H. Holstein-Rathlou, "Parallel computer simulation of nearest-neighbour interaction in a system of nephrons," Parallel Comput. 12, 113-120 (1989).

${ }^{10}$ R. C. Elson, H. I. Selverston, R. Huerta, N. R. Rulkov, M. I. Rabinovich, and H. D. I. Abarbanel, "Synchronous behavior of two coupled biological neurons,” Phys. Rev. Lett. 81, 5692-5695 (1998).
${ }^{11}$ G.-I. Bischi, L. Stefanini, and L. Gardini, "Synchronization, intermittency, and critical curves in a duopoly game," Math. Comput. Simul. 44, 559585 (1998).

${ }^{12}$ P. M. Dean and E. K. Matthews, "Glucose induced electrical activity in pancreatic islet cells," J. Physiol. (London) 210, 255-264 (1970).

${ }^{13}$ E. Mosekilde, B. Lading, S. Yanchuk, and Yu. Maistrenko, "Bifurcation structure of a model of bursting pancreatic cells," BioSystems 63, 3-13 (2001).

${ }^{14}$ R. M. Santos, L. M. Rosario, A. Nadal, J. Garcia-Sancho, B. Soria, and M. Valdeolmillos, "Widespread synchronous $\left[\mathrm{Ca}^{2+}\right]_{i}$ oscillations due to bursting electrical activity in single pancreatic islets," Eur. J. Physiol. 418, 417-422 (1991).

${ }^{15}$ C. Haxholdt, C. Kampmann, E. Mosekilde, and J. D. Sterman, "Entrainment in a disaggregated economic long wave model," Syst. Dyn. Rev. 11, 177-198 (1995).

${ }^{16}$ K. Pyragas, "Weak and strong synchronization of chaos," Phys. Rev. E 54, R4508-R4511 (1996).

${ }^{17} \mathrm{~K}$. Kaneko, "Lyapunov analysis and information flow in coupled map lattices," Physica D 23, 436-447 (1986).

${ }^{18} \mathrm{~K}$. Kaneko, "Relevance of dynamic clustering in biological networks," Physica D 75, 55-73 (1994).

${ }^{19}$ O. Popovych, Yu. Maistrenko, and E. Mosekilde, "Cluster formation and cluster splitting in a system of globally coupled maps," in Emergent $\mathrm{Na}$ ture, edited by M. M. Novac (World Scientific, Singapore, 2002), pp. $325-334$.

${ }^{20}$ J. C. Alexander, J. A. Yorke, Z. You, and I. Kan, "Riddled basins," Int. J. Bifurcation Chaos Appl. Sci. Eng. 2, 795-813 (1992).

${ }^{21}$ Yu. L. Maistrenko, V. L. Maistrenko, O. Popovych, and E. Mosekilde, "Unfolding the riddling bifurcation," Phys. Lett. A 262, 355-360 (1999).

${ }^{22} \mathrm{E}$. Ott and J. C. Sommerer, "Blowout bifurcations: The occurrence of riddled basins and on-off intermittency," Phys. Lett. A 188, 39-47 (1994).

${ }^{23} \mathrm{H}$. Fujisaka and T. Yamada, "A new intermittency in coupled dynamical systems," Prog. Theor. Phys. 74, 918 (1985).

${ }^{24}$ A. S. Pikovsky, "On the interaction of strange attractors," Z. Phys. B: Condens. Matter 55, 149 (1984).

${ }^{25}$ T. Kapitaniak, "Transition to hyperchaos in chaotically forced coupled oscillators," Phys. Rev. E 47, R2975-R2978 (1993).

${ }^{26} \mathrm{~S}$. Yanchuk and T. Kapitaniak, "Symmetry increasing bifurcation as a predictor of chaos-hyperchaos transition in coupled systems," Phys. Rev. E 64, 056235 (2001).

${ }^{27}$ S. Yanchuk, Yu. Maistrenko, and E. Mosekilde, "Loss of synchronization in coupled Rössler systems," Physica D 154, 26-42 (2001).

${ }^{28}$ S. Yanchuk, Yu. Maistrenko, and E. Mosekilde, "Partial synchronization and clustering in a system of diffusively coupled chaotic oscillators," Math. Comput. Simul. 54, 491-508 (2001).

${ }^{29}$ S. Yanchuk, Yu. Maistrenko, B. Lading, and E. Mosekilde, "Effects of a parameter mismatch on the synchronization of two coupled chaotic oscillators," Int. J. Bifurcation Chaos Appl. Sci. Eng. 10, 2629-2648 (2000).

${ }^{30}$ B. Lading, E. Mosekilde, S. Yanchuk, and Yu. Maistrenko, "Chaotic synchronization between coupled pancreatic beta-cells," Prog. Theor. Phys. Suppl. 139, 164-177 (2000).

${ }^{31}$ J. F. Heagy, T. L. Carroll, and L. M. Pecora, "Desynchronization by periodic orbits," Phys. Rev. E 52, R1253-R1256 (1995).

${ }^{32}$ B. R. Hunt, "Optimal periodic orbits of chaotic systems," Phys. Rev. Lett. 76, 2254-2257 (1996).

${ }^{33}$ A. Sherman and J. Rinzel, "Model for synchronization of pancreatic $\beta$-cells by gap junction coupling," Biophys. J. 59, 547-559 (1991).

${ }^{34}$ J. Milnor, "On the concept of attractor," Commun. Math. Phys. 99, 177195 (1985).

${ }^{35}$ D. J. Gauthier and J. C. Bienfang, "Intermittent loss of synchronization in coupled chaotic oscillators: Toward a new criterion for high-quality synchronization," Phys. Rev. Lett. 77, 1751-1754 (1996).

${ }^{36}$ N. F. Rul'kov and M. M. Sushchik, "Robustness of synchronized chaotic oscillations,” Int. J. Bifurcation Chaos Appl. Sci. Eng. 7, 625-643 (1997).

${ }^{37}$ C. Grebogi, E. Ott, and J. A. Yorke, "Unstable periodic orbits and the dimensions of multifractal chaotic attractors," Phys. Rev. A 37, 17111724 (1988).

${ }^{38}$ Y.-C. Lai, Y. Nagai, and C. Grebogi, "Characterization of the natural measure by unstable periodic orbits in chaotic attractors," Phys. Rev. Lett. 79, 649-652 (1997)

${ }^{39}$ Y. Nagai and Y.-C. Lai, "Characterization of blowout bifurcation by unstable periodic orbits,” Phys. Rev. E 55, R1251-R1254 (1997). 
${ }^{40}$ Y. Nagai and Y.-C. Lai, "Periodic-orbit theory of the blowout bifurcation," Phys. Rev. E 56, 4031-4041 (1997).

${ }^{41}$ Y.-C. Lai, "Unstable dimension variability and complexity in chaotic systems," Phys. Rev. E 59, R3807-3810 (1999).

${ }^{42}$ Yu. L. Maistrenko, V. L. Maistrenko, A. Popovich, and E. Mosekilde, "Transverse instability and riddled basins in a system of two coupled logistic maps," Phys. Rev. E 57, 2713-2724 (1998).

${ }^{43}$ G. A. Johnson, D. J. Mar, T. L. Carroll, and L. M. Pecora, "Synchronization and imposed bifurcations in the presence of large parameter mismatch,” Phys. Rev. Lett. 80, 3956-3959 (1998).
${ }^{44}$ O. Popovych, Yu. L. Maistrenko, E. Mosekilde, A. S. Pikovsky, and J. Kurths, "Transcritical loss of synchronization in coupled chaotic systems," Phys. Lett. A 275, 401 (2000).

${ }^{45}$ V. N. Belykh, I. V. Belykh, and E. Mosekilde, "Cluster synchronization modes in an ensemble of coupled chaotic oscillators," Phys. Rev. E 63, 036216 (2001).

${ }^{46} \mathrm{~S}$. Yanchuk, "Preserving of one-sided invariance in $R^{n}$ with respect to systems of ordinary differential equations," Nonlinear Oscillations 2, 278-285 (2002) 